Supporting Information

\title{
An Efficient Method for Calculating Atomic Charges of Peptides and Proteins from Electronic Populations
}

\author{
Young Kee Kang ${ }^{\dagger}$ and Harold A. Scheraga** \\ Department of Chemistry, Chungbuk National University, Cheongju, Chungbuk 361-763, Republic of Korea, \\ and Baker Laboratory of Chemistry and Chemical Biology, Cornell University, Ithaca, New York 14853-1301
}

* To whom correspondence should be addressed. Telephone: (607) 255-4034. Fax: (607) 254-4700.

E-mail: has5@cornell.edu.

${ }^{\dagger}$ Chungbuk National University. ${ }^{*}$ Cornell University. 
TABLE S1: Atomic Charges for the Extended Conformations of Ac-Ala-NHMe and Ac-(Ala) ${ }_{3}$-NHMe Calculated by the B3LYP/6-31G(d,p), EPAC, and Electrostatic Potential (ESP)-Fitted Methods ${ }^{a}$

\begin{tabular}{|c|c|c|c|c|c|c|c|c|c|c|c|c|}
\hline \multirow[b]{3}{*}{ atom } & \multicolumn{4}{|c|}{ Mulliken population $^{b}$} & \multicolumn{4}{|c|}{ EPAC } & \multicolumn{4}{|c|}{ ESP-fitted $^{b}$} \\
\hline & \multirow[b]{2}{*}{ Ala } & \multicolumn{3}{|c|}{$(\text { Ala })_{3}$} & \multirow[b]{2}{*}{ Ala } & \multicolumn{3}{|c|}{$(\text { Ala })_{3}$} & \multirow[b]{2}{*}{ Ala } & \multicolumn{3}{|c|}{$(\text { Ala })_{3}$} \\
\hline & & Ala1 & Ala2 & Ala3 & & Ala1 & Ala2 & Ala3 & & Ala1 & Ala2 & Ala3 \\
\hline $\mathrm{N}$ & -0.519 & -0.520 & -0.530 & -0.530 & -0.515 & -0.515 & -0.517 & -0.517 & -0.607 & -0.723 & -0.706 & -0.647 \\
\hline $\mathrm{H}$ & 0.269 & 0.268 & 0.276 & 0.278 & 0.276 & 0.276 & 0.275 & 0.275 & 0.308 & 0.340 & 0.319 & 0.308 \\
\hline $\mathrm{C}^{\alpha}$ & 0.004 & 0.006 & 0.000 & -0.004 & -0.005 & -0.005 & -0.006 & -0.005 & 0.173 & 0.349 & 0.376 & 0.269 \\
\hline $\mathrm{H}^{\alpha}$ & 0.123 & 0.130 & 0.133 & 0.127 & 0.139 & 0.139 & 0.139 & 0.139 & 0.027 & 0.020 & 0.025 & 0.013 \\
\hline $\mathrm{C}^{\beta}$ & -0.305 & -0.310 & -0.310 & -0.306 & -0.295 & -0.295 & -0.295 & -0.295 & -0.247 & -0.348 & -0.383 & -0.294 \\
\hline $\mathrm{C}$ & 0.585 & 0.592 & 0.595 & 0.589 & 0.578 & 0.576 & 0.576 & 0.577 & 0.503 & 0.568 & 0.542 & 0.492 \\
\hline $\mathrm{O}$ & -0.521 & -0.537 & -0.535 & -0.519 & -0.521 & -0.517 & -0.517 & -0.521 & -0.481 & -0.527 & -0.497 & -0.476 \\
\hline $\mathrm{H}^{\beta}$ & 0.097 & 0.104 & 0.109 & 0.102 & 0.115 & 0.115 & 0.115 & 0.115 & 0.038 & 0.069 & 0.082 & 0.049 \\
\hline $\mathrm{H}^{\beta}$ & 0.143 & 0.143 & 0.143 & 0.144 & 0.115 & 0.115 & 0.115 & 0.115 & 0.105 & 0.121 & 0.127 & 0.113 \\
\hline $\mathrm{H}^{\beta}$ & 0.116 & 0.113 & 0.117 & 0.122 & 0.115 & 0.115 & 0.115 & 0.115 & 0.084 & 0.097 & 0.110 & 0.096 \\
\hline total charge & -0.008 & -0.011 & -0.003 & 0.002 & 0.001 & 0.003 & 0.000 & -0.002 & -0.097 & -0.034 & -0.005 & -0.078 \\
\hline
\end{tabular}

${ }^{a}$ Charges in electronic charges e. ${ }^{b}$ Computed at the B3LYP/6-31G(d,p)//HF/6-31G(d,p) level. 
TABLE S2: Comparison of Atomic Charges and Dipole Moments for Conformation E of Ac-Ala-NHMe Calculated by the B3LYP/6-31G(d,p), EPAC, and GDAC Methods ${ }^{a}$

\begin{tabular}{|c|c|c|c|c|c|c|}
\hline residue & atom & B3LYP & EPAC & $\operatorname{abs} \Delta^{b}$ & GDAC & $\operatorname{abs} \Delta^{c}$ \\
\hline \multirow[t]{6}{*}{ Ac } & $\mathrm{H}_{\mathrm{Me}}$ & 0.129 & 0.130 & 0.001 & 0.073 & 0.056 \\
\hline & $\mathrm{H}_{\mathrm{Me}}$ & 0.129 & 0.130 & 0.001 & 0.073 & 0.056 \\
\hline & $\mathrm{H}_{\mathrm{Me}}$ & 0.129 & 0.130 & 0.001 & 0.073 & 0.056 \\
\hline & $\mathrm{C}_{\mathrm{Me}}$ & -0.392 & -0.397 & 0.005 & -0.092 & 0.300 \\
\hline & $\mathrm{O}$ & -0.515 & -0.521 & 0.006 & -0.296 & 0.219 \\
\hline & $\mathrm{C}$ & 0.565 & 0.563 & 0.002 & 0.018 & 0.547 \\
\hline \multirow[t]{10}{*}{ Ala } & $\mathrm{N}$ & -0.519 & -0.515 & 0.004 & -0.247 & 0.272 \\
\hline & $\mathrm{H}$ & 0.269 & 0.276 & 0.007 & 0.266 & 0.003 \\
\hline & $\mathrm{C}^{\alpha}$ & 0.004 & -0.005 & 0.009 & 0.135 & 0.131 \\
\hline & $\mathrm{H}^{\alpha}$ & 0.123 & 0.139 & 0.016 & 0.095 & 0.028 \\
\hline & $\mathrm{C}^{\beta}$ & -0.305 & -0.295 & 0.010 & -0.163 & 0.142 \\
\hline & $\mathrm{C}$ & 0.585 & 0.578 & 0.007 & 0.020 & 0.565 \\
\hline & $\mathrm{O}$ & -0.521 & -0.521 & 0.000 & -0.294 & 0.227 \\
\hline & $\mathrm{H}^{\beta}$ & 0.119 & 0.115 & 0.004 & 0.062 & 0.057 \\
\hline & $\mathrm{H}^{\beta}$ & 0.119 & 0.115 & 0.004 & 0.062 & 0.057 \\
\hline & $\mathrm{H}^{\beta}$ & 0.119 & 0.115 & 0.004 & 0.062 & 0.057 \\
\hline \multirow[t]{6}{*}{ NHMe } & $\mathrm{N}$ & -0.525 & -0.524 & 0.001 & -0.265 & 0.261 \\
\hline & $\mathrm{H}$ & 0.264 & 0.276 & 0.012 & 0.269 & 0.005 \\
\hline & $\mathrm{C}_{\mathrm{Me}}$ & -0.167 & -0.167 & 0.000 & -0.068 & 0.099 \\
\hline & $\mathrm{H}_{\mathrm{Me}}$ & 0.130 & 0.127 & 0.003 & 0.072 & 0.058 \\
\hline & $\mathrm{H}_{\mathrm{Me}}$ & 0.130 & 0.126 & 0.004 & 0.072 & 0.058 \\
\hline & $\mathrm{H}_{\mathrm{Me}}$ & 0.130 & 0.127 & 0.003 & 0.072 & 0.058 \\
\hline & & & \multirow{2}{*}{$\begin{array}{l}\operatorname{mad}^{d} \\
\operatorname{rmsd}^{e}\end{array}$} & 0.005 & \multirow{2}{*}{$\begin{array}{l}\operatorname{mad}^{d} \\
\mathrm{rmsd}^{e}\end{array}$} & 0.150 \\
\hline & & & & 0.006 & & 0.216 \\
\hline & $\mu$ & 2.92 & 2.95 & & 2.41 & \\
\hline
\end{tabular}

${ }^{a}$ Charges in electronic charges e and dipole moments in Debyes. ${ }^{b}$ Absolute deviations between atomic charges calculated at the B3LYP/6-31G(d,p)//HF/6-31G(d,p) level and by the EPAC method. ${ }^{c}$ Absolute deviations between atomic charges calculated at the $\mathrm{B} 3 \mathrm{LYP} / 6-31 \mathrm{G}(\mathrm{d}, \mathrm{p}) / / \mathrm{HF} / 6-31 \mathrm{G}(\mathrm{d}, \mathrm{p})$ level and by the GDAC method. ${ }^{d}$ Mean absolute deviation. ${ }^{e}$ Root mean square deviation. 
TABLE S3: Comparison of Mulliken and ESP-fitted Charges for Conformations C, E, and D of Ac-Ala-NHMe Calculated at the B3LYP/6-31G(d,p)//HF/6-31G(d,p) Level ${ }^{a}$

\begin{tabular}{|c|c|c|c|c|c|c|c|}
\hline \multirow[b]{2}{*}{ residue } & \multirow[b]{2}{*}{ atom } & \multicolumn{3}{|c|}{ Mulliken charges } & \multicolumn{3}{|c|}{ ESP-fitted charges } \\
\hline & & $\mathrm{C}$ & $\mathrm{E}$ & $\mathrm{D}$ & $\mathrm{C}$ & $\mathrm{E}$ & $\mathrm{D}$ \\
\hline \multirow[t]{6}{*}{ Ac } & $\mathrm{H}_{\mathrm{Me}}$ & 0.150 & 0.142 & 0.149 & 0.185 & 0.146 & 0.146 \\
\hline & $\mathrm{H}_{\mathrm{Me}}$ & 0.147 & 0.141 & 0.142 & 0.145 & 0.151 & 0.152 \\
\hline & $\mathrm{H}_{\mathrm{Me}}$ & 0.103 & 0.104 & 0.111 & 0.176 & 0.130 & 0.127 \\
\hline & $\mathrm{C}_{\mathrm{Me}}$ & -0.391 & -0.392 & -0.405 & -0.625 & -0.531 & -0.532 \\
\hline & $\mathrm{O}$ & -0.520 & -0.515 & -0.481 & -0.566 & -0.550 & -0.546 \\
\hline & $\mathrm{C}$ & 0.571 & 0.565 & 0.554 & 0.743 & 0.727 & 0.802 \\
\hline \multirow[t]{10}{*}{ Ala } & $\mathrm{N}$ & -0.504 & -0.519 & -0.539 & -0.584 & -0.607 & -0.804 \\
\hline & $\mathrm{H}$ & 0.259 & 0.269 & 0.254 & 0.333 & 0.308 & 0.350 \\
\hline & $\mathrm{C}^{\alpha}$ & -0.026 & 0.004 & -0.025 & 0.186 & 0.173 & 0.303 \\
\hline & $\mathrm{H}^{\alpha}$ & 0.132 & 0.123 & 0.169 & 0.037 & 0.027 & 0.046 \\
\hline & $C^{\beta}$ & -0.322 & -0.305 & -0.307 & -0.371 & -0.247 & -0.280 \\
\hline & $\mathrm{C}$ & 0.594 & 0.585 & 0.586 & 0.529 & 0.503 & 0.503 \\
\hline & $\mathrm{O}$ & -0.516 & -0.521 & -0.516 & -0.498 & -0.481 & -0.504 \\
\hline & $\mathrm{H}^{\beta}$ & 0.127 & 0.143 & 0.145 & 0.109 & 0.038 & 0.071 \\
\hline & $\mathrm{H}^{\beta}$ & 0.104 & 0.097 & 0.099 & 0.107 & 0.105 & 0.089 \\
\hline & $\mathrm{H}^{\beta}$ & 0.126 & 0.116 & 0.109 & 0.106 & 0.084 & 0.075 \\
\hline \multirow[t]{6}{*}{ NHMe } & $\mathrm{N}$ & -0.524 & -0.525 & -0.523 & -0.470 & -0.388 & -0.418 \\
\hline & $\mathrm{H}$ & 0.282 & 0.264 & 0.263 & 0.323 & 0.305 & 0.298 \\
\hline & $\mathrm{C}_{\mathrm{Me}}$ & -0.167 & -0.167 & -0.165 & -0.162 & -0.309 & -0.228 \\
\hline & $\mathrm{H}_{\mathrm{Me}}$ & 0.142 & 0.148 & 0.128 & 0.107 & 0.149 & 0.127 \\
\hline & $\mathrm{H}_{\mathrm{Me}}$ & 0.121 & 0.132 & 0.146 & 0.093 & 0.131 & 0.113 \\
\hline & $\mathrm{H}_{\mathrm{Me}}$ & 0.109 & 0.111 & 0.105 & 0.098 & 0.137 & 0.111 \\
\hline
\end{tabular}

${ }^{a}$ Charges in electronic charges e. Conformations C, E, and D are presented in Table S4 of the Supporting Information. 
TABLE S4: Torsion Angles, Relative Conformational Energies, and Dipole Moments of Representative Conformations for AcX-NHMe Calculated at the B3LYP/6-31G(d,p)//HF/6-31G(d,p) Level and Their Dipole Moments Computed by the EPAC $\operatorname{Method~}^{a}$

\section{(1) Ac-Ala-NHMe}

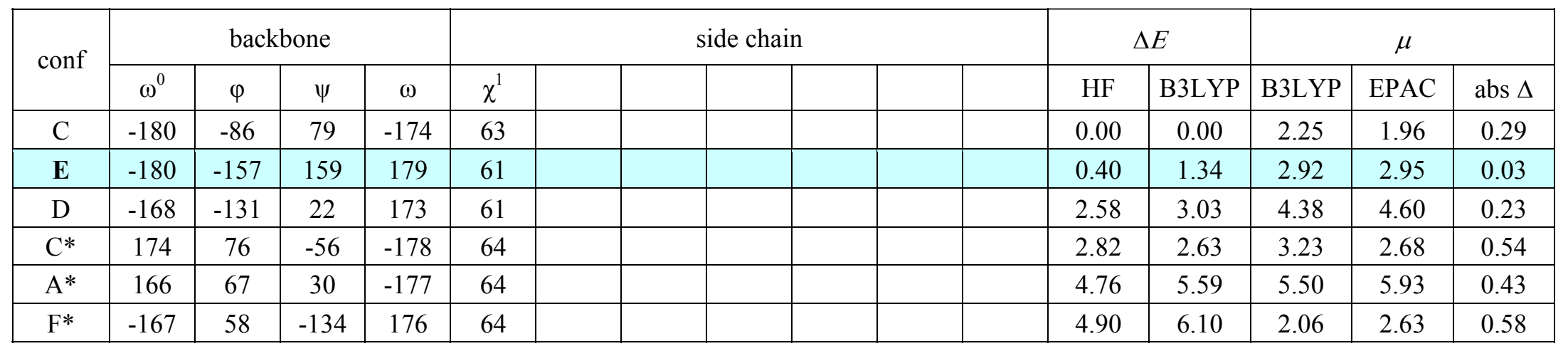

\section{(2) Ac-Val-NHMe}

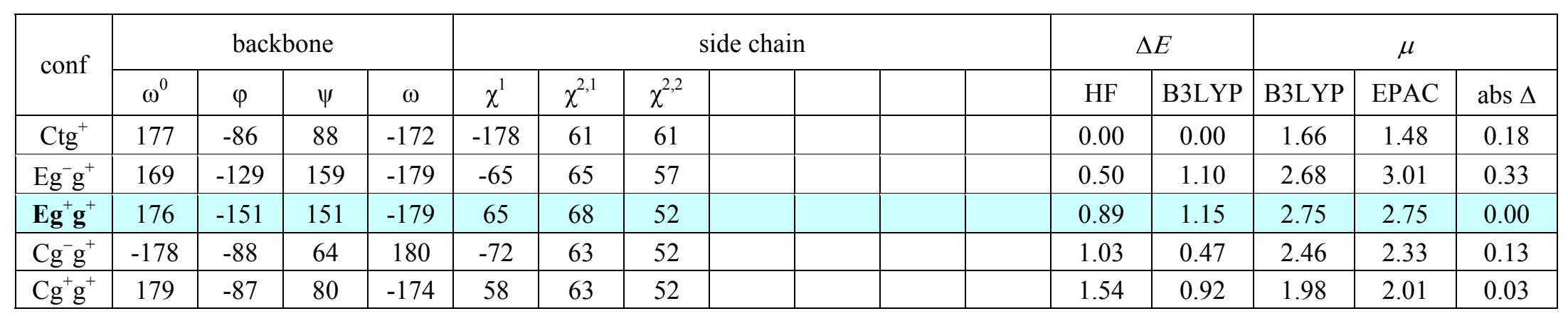


(3) Ac-Leu-NHMe

\begin{tabular}{|c|c|c|c|c|c|c|c|c|c|c|c|c|c|}
\hline \multirow{2}{*}{ conf } & \multicolumn{4}{|c|}{ backbone } & \multicolumn{6}{|c|}{ side chain } & \multicolumn{3}{|c|}{$\mu$} \\
\hline & $\omega^{0}$ & $\varphi$ & $\psi$ & $\omega$ & $\chi^{1}$ & $\chi^{2}$ & $\chi^{3,1}$ & $\chi^{3,2}$ & $\mathrm{HF}$ & B3LYP & B3LYP & EPAC & abs $\Delta$ \\
\hline $\mathrm{Ctg}^{+}$ & 179 & -86 & 84 & -173 & -174 & 63 & 58 & 58 & 0.00 & 0.00 & 1.83 & 1.81 & 0.03 \\
\hline $\mathrm{Cg}^{-} \mathrm{t}$ & 179 & -87 & 81 & -174 & -60 & 176 & 63 & 64 & 0.19 & 0.25 & 2.15 & 1.81 & 0.33 \\
\hline $\mathbf{E g}^{-} \mathbf{t}$ & 171 & -128 & 151 & 179 & -63 & 171 & 62 & 64 & 0.44 & 1.07 & 2.27 & 2.72 & 0.45 \\
\hline $\mathrm{Ctt}$ & 178 & -85 & 87 & -172 & -155 & 177 & 64 & 64 & 1.19 & 0.91 & 1.75 & 1.70 & 0.05 \\
\hline $\operatorname{Etg}^{+}$ & -177 & -152 & 138 & -180 & -176 & 64 & 58 & 58 & 1.32 & 2.13 & 2.49 & 2.42 & 0.07 \\
\hline $\mathrm{Cg}^{-} \mathrm{g}^{+}$ & -177 & -87 & 78 & -174 & -83 & 63 & 58 & 56 & 1.89 & 1.62 & 2.21 & 1.95 & 0.25 \\
\hline $\mathrm{Eg}^{+} \mathrm{g}^{+}$ & 173 & -151 & 168 & -179 & 55 & 72 & 57 & 58 & 1.98 & 2.24 & 2.90 & 3.11 & 0.21 \\
\hline $\mathrm{Ctg}^{-}$ & 178 & -85 & 88 & -172 & -157 & -59 & 69 & 53 & 2.12 & 1.71 & 1.71 & 1.68 & 0.04 \\
\hline $\mathrm{Eg}^{+} \mathrm{t}$ & 178 & -155 & 164 & -180 & 76 & 174 & 63 & 67 & 2.17 & 2.65 & 2.99 & 3.07 & 0.08 \\
\hline $\mathrm{Cg}^{-} \mathrm{g}^{-}$ & 180 & -86 & 82 & -173 & -70 & -55 & 71 & 50 & 2.69 & 2.61 & 2.06 & 1.76 & 0.30 \\
\hline Ett & -177 & -151 & 135 & -179 & -177 & 146 & 60 & 56 & 3.01 & 3.63 & 2.36 & 2.31 & 0.06 \\
\hline $\mathrm{Eg}^{-} \mathrm{g}^{-}$ & 169 & -122 & 148 & 179 & -74 & -58 & 71 & 48 & 3.01 & 3.42 & 2.26 & 2.73 & 0.47 \\
\hline $\mathrm{Cg}^{+} \mathrm{g}^{+}$ & -173 & -87 & 48 & 173 & 44 & 56 & 58 & 55 & 3.75 & 2.86 & 2.98 & 2.98 & 0.01 \\
\hline $\mathrm{Etg}^{-}$ & -175 & -145 & 121 & -177 & -172 & -74 & 71 & 49 & 3.88 & 4.48 & 1.86 & 1.82 & 0.04 \\
\hline $\mathrm{Cg}^{+} \mathrm{t}$ & -177 & -87 & 79 & -174 & 74 & -176 & 65 & 67 & 4.12 & 3.13 & 2.00 & 2.08 & 0.07 \\
\hline $\mathrm{Eg}^{+} \mathrm{g}^{-}$ & 177 & -152 & 160 & 177 & 69 & -56 & 72 & 50 & 4.46 & 4.52 & 2.90 & 3.06 & 0.16 \\
\hline
\end{tabular}


(4) Ac-Ile-NHMe

\begin{tabular}{|c|c|c|c|c|c|c|c|c|c|c|c|c|c|}
\hline \multirow{2}{*}{ conf } & \multicolumn{4}{|c|}{ backbone } & \multicolumn{6}{|c|}{ side chain } & \multicolumn{3}{|c|}{$\mu$} \\
\hline & $\omega^{0}$ & $\varphi$ & $\psi$ & $\omega$ & $\chi^{1}$ & $\chi^{2,1}$ & $\chi^{2,2}$ & $\chi^{3,1}$ & $\mathrm{HF}$ & B3LYP & B3LYP & EPAC & abs $\Delta$ \\
\hline $\mathrm{Cg}^{-} \mathrm{t}$ & 176 & -86 & 90 & -172 & -56 & 173 & 63 & 66 & 0.00 & 0.00 & 1.58 & 1.38 & 0.20 \\
\hline $\mathrm{Cg}^{-} \mathrm{g}^{-}$ & 176 & -87 & 88 & -172 & -52 & -59 & 58 & 63 & 0.21 & 0.26 & 1.68 & 1.47 & 0.21 \\
\hline $\mathrm{Eg}^{+} \mathrm{t}$ & 169 & -127 & 160 & -179 & 60 & 170 & 68 & 65 & 0.35 & 0.96 & 2.67 & 3.04 & 0.36 \\
\hline Ett & 176 & -151 & 152 & -179 & -166 & 165 & 71 & 65 & 0.54 & 0.78 & 2.79 & 2.76 & 0.03 \\
\hline $\mathrm{Cg}^{+} \mathrm{t}$ & -176 & -87 & 63 & 179 & 53 & 157 & 61 & 59 & 0.58 & 0.17 & 2.52 & 2.40 & 0.12 \\
\hline $\mathrm{Etg}^{+}$ & 179 & -152 & 153 & -179 & -166 & 61 & 70 & 52 & 0.96 & 1.20 & 2.69 & 2.70 & 0.01 \\
\hline $\mathrm{Ctg}^{+}$ & 179 & -87 & 82 & -174 & -178 & 56 & 62 & 52 & 1.26 & 0.65 & 1.80 & 1.92 & 0.12 \\
\hline $\mathrm{Ctt}$ & 179 & -87 & 79 & -174 & -175 & 163 & 64 & 65 & 1.30 & 0.71 & 1.90 & 2.01 & 0.11 \\
\hline $\mathrm{Eg}^{+} \mathrm{g}^{+}$ & 168 & -125 & 165 & -178 & 56 & 74 & 68 & 53 & 1.90 & 2.06 & 2.76 & 3.24 & 0.48 \\
\hline $\mathrm{Eg}^{+} \mathrm{g}^{-}$ & 169 & -129 & 159 & -179 & 75 & -63 & 67 & 70 & 2.39 & 2.68 & 2.71 & 3.06 & 0.35 \\
\hline $\mathrm{Eg}^{-} \mathrm{g}^{+}$ & 174 & -117 & 124 & -177 & -55 & 100 & 63 & 60 & 2.55 & 3.19 & 1.40 & 1.56 & 0.17 \\
\hline $\mathrm{Bg}^{+} \mathrm{g}^{+}$ & -173 & -87 & 47 & 173 & 45 & 55 & 61 & 55 & 2.60 & 1.83 & 2.93 & 2.95 & 0.03 \\
\hline $\mathrm{Etg}^{-}$ & -179 & -147 & 135 & -179 & -171 & -87 & 62 & 58 & 3.62 & 3.81 & 2.34 & 2.24 & 0.09 \\
\hline $\mathrm{Ctg}^{-}$ & 177 & -86 & 91 & -172 & -162 & -66 & 67 & 68 & 3.75 & 3.23 & 1.52 & 1.68 & 0.16 \\
\hline $\mathrm{Cg}^{+} \mathrm{g}^{-}$ & -177 & -88 & 78 & -174 & 71 & -55 & 71 & 71 & 3.96 & 2.85 & 2.06 & 2.02 & 0.04 \\
\hline
\end{tabular}

\section{(5) Ac-Gly-NHMe}

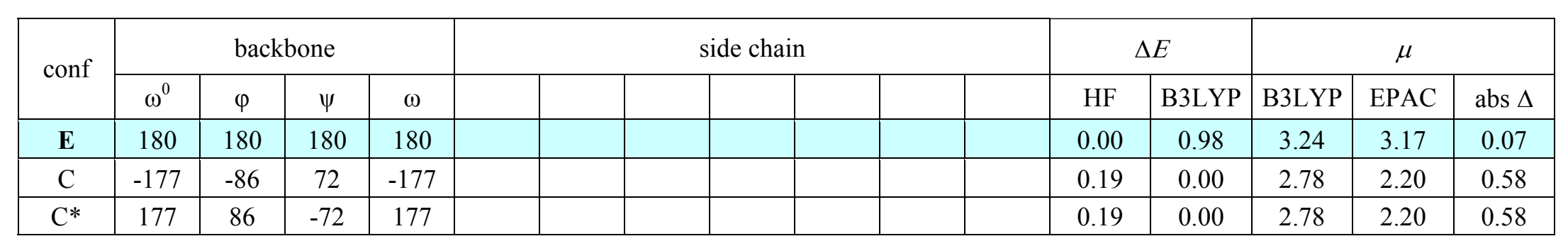


(6) Ac-Pro-NHMe

\begin{tabular}{|c|c|c|c|c|c|c|c|c|c|c|c|c|c|c|}
\hline \multirow{2}{*}{ conf } & \multicolumn{4}{|c|}{ backbone } & \multicolumn{5}{|c|}{ side chain } & \multicolumn{2}{|c|}{$\Delta E$} & \multicolumn{3}{|c|}{$\mu$} \\
\hline & $\omega^{0}$ & $\varphi$ & $\psi$ & $\omega$ & $\chi^{0}$ & $\chi^{1}$ & $\chi^{2}$ & $\chi^{3}$ & $\chi^{4}$ & $\mathrm{HF}$ & B3LYP & B3LYP & EPAC & abs $\Delta$ \\
\hline tCd & -173 & -86 & 75 & -176 & -15 & 32 & -38 & 29 & -9 & 0.00 & 0.00 & 2.65 & 2.80 & 0.15 \\
\hline $\mathrm{tCu}$ & -175 & -83 & 84 & -174 & -11 & -14 & 31 & -37 & 30 & 1.89 & 1.18 & 2.69 & 2.62 & 0.07 \\
\hline $\mathrm{cAd}$ & 11 & -91 & -4 & -178 & -13 & 32 & -38 & 30 & -10 & 3.01 & 4.28 & 1.52 & 2.07 & 0.55 \\
\hline $\mathrm{cAu}$ & 8 & -77 & -20 & -174 & 3 & -25 & 37 & -35 & 20 & 4.19 & 5.33 & 2.14 & 2.75 & 0.61 \\
\hline $\mathrm{tAu}$ & -171 & -72 & -21 & 176 & 6 & -27 & 38 & -33 & 17 & 4.42 & 6.07 & 5.68 & 6.32 & 0.64 \\
\hline
\end{tabular}

\section{(7) Ac-Cys-NHMe}

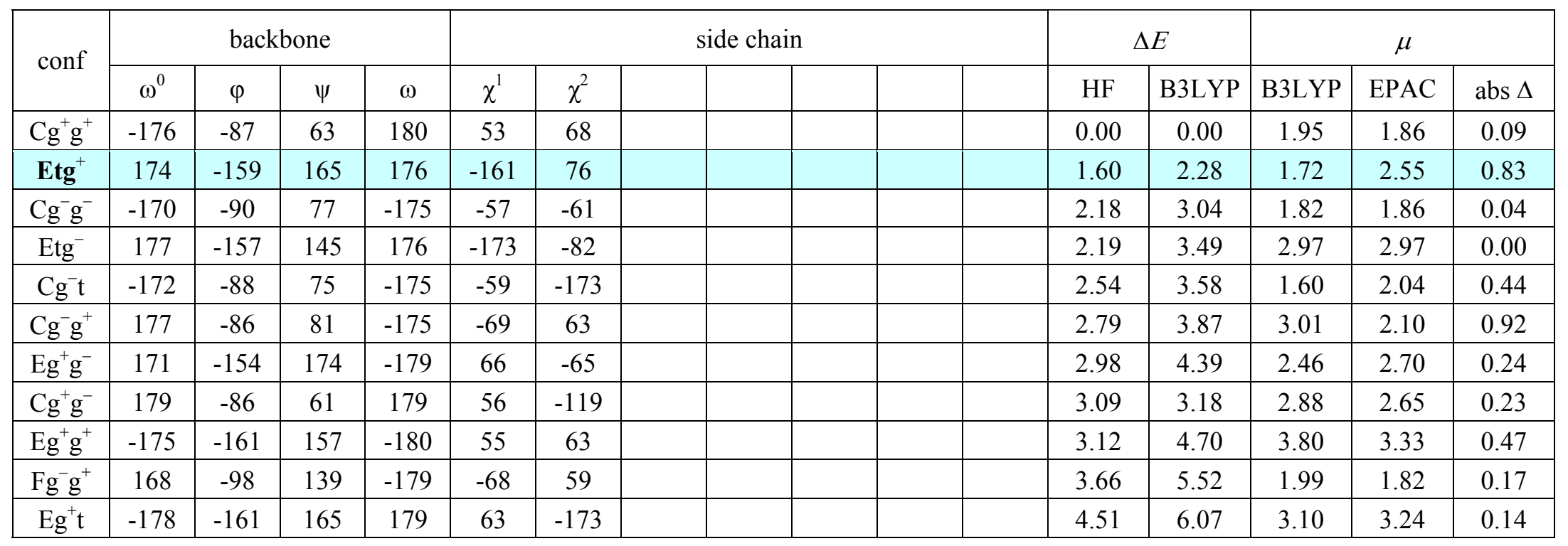


(8) Ac-Met-NHMe

\begin{tabular}{|c|c|c|c|c|c|c|c|c|c|c|c|c|c|}
\hline \multirow{2}{*}{ conf } & \multicolumn{4}{|c|}{ backbone } & \multicolumn{6}{|c|}{ side chain } & \multicolumn{3}{|c|}{$\mu$} \\
\hline & $\omega^{0}$ & $\varphi$ & $\psi$ & $\omega$ & $\chi^{1}$ & $\chi^{2}$ & $x^{3}$ & $\chi^{4}$ & $\mathrm{HF}$ & B3LYP & B3LYP & EPAC & abs $\Delta$ \\
\hline $\mathrm{Etg}^{+}$ & 178 & -157 & 150 & 180 & -175 & 52 & 56 & 57 & 0.00 & 0.00 & 1.17 & 2.33 & 1.17 \\
\hline $\mathrm{Ctt}$ & -179 & -86 & 81 & -174 & -173 & 174 & 175 & 180 & 1.26 & 2.16 & 1.55 & 1.26 & 0.29 \\
\hline $\mathrm{Ctg}^{+}$ & -177 & -86 & 80 & -174 & -175 & 67 & -177 & -180 & 1.40 & 1.87 & 1.70 & 1.64 & 0.06 \\
\hline $\mathrm{Eg}^{-} \mathrm{g}^{-}$ & 169 & -136 & 157 & 179 & -77 & -75 & 138 & -177 & 1.46 & 2.08 & 1.15 & 2.28 & 1.13 \\
\hline $\mathrm{Cg}^{+} \mathrm{g}^{-}$ & -175 & -87 & 62 & 179 & 60 & -83 & 175 & -180 & 1.65 & 0.77 & 2.08 & 2.14 & 0.06 \\
\hline $\mathrm{Cg}^{-} \mathrm{t}$ & 177 & -86 & 81 & -174 & -66 & 179 & 179 & 180 & 1.65 & 2.50 & 3.23 & 2.27 & 0.96 \\
\hline $\mathrm{Eg}^{-} \mathrm{t}$ & 170 & -135 & 155 & 179 & -63 & -177 & -179 & -180 & 1.67 & 3.20 & 1.82 & 2.22 & 0.40 \\
\hline $\mathrm{Eg}^{+} \mathrm{t}$ & 174 & -153 & 164 & -180 & 62 & -179 & 180 & 180 & 1.75 & 3.25 & 3.38 & 3.11 & 0.28 \\
\hline $\mathrm{Cg}^{-} \mathrm{g}^{+}$ & -177 & -86 & 77 & -175 & -56 & 89 & 76 & -61 & 1.78 & 1.40 & 1.47 & 1.79 & 0.32 \\
\hline $\mathrm{Cg}^{-} \mathrm{g}^{-}$ & 178 & -85 & 80 & -174 & -66 & -68 & -74 & 65 & 1.97 & 2.25 & 1.93 & 1.90 & 0.03 \\
\hline $\mathrm{Cg}^{+} \mathrm{t}$ & -175 & -87 & 62 & 179 & 56 & 173 & -76 & -57 & 2.60 & 2.69 & 2.18 & 2.15 & 0.03 \\
\hline $\mathrm{Ctg}^{-}$ & 177 & -86 & 84 & -175 & -150 & -58 & -68 & 67 & 2.61 & 2.32 & 2.88 & 1.98 & 0.91 \\
\hline $\mathrm{Etg}^{-}$ & -176 & -150 & 112 & 176 & -164 & -64 & -63 & -54 & 3.64 & 4.24 & 1.51 & 1.63 & 0.12 \\
\hline $\mathrm{Eg}^{+} \mathrm{g}^{+}$ & 173 & -155 & 161 & 172 & 64 & 96 & -170 & -179 & 3.68 & 4.44 & 1.97 & 2.67 & 0.70 \\
\hline $\mathrm{Eg}^{+} \mathrm{g}^{-}$ & -176 & -153 & 154 & -180 & 66 & -76 & -172 & 180 & 3.73 & 4.83 & 4.05 & 3.43 & 0.62 \\
\hline $\mathrm{Eg}^{-} \mathrm{g}^{+}$ & -179 & -142 & 147 & 180 & -61 & 92 & -178 & -179 & 3.80 & 5.10 & 2.95 & 2.69 & 0.26 \\
\hline
\end{tabular}

\section{(9) $\mathrm{Ac}-\mathrm{His}(\delta)-\mathrm{NHMe}$}

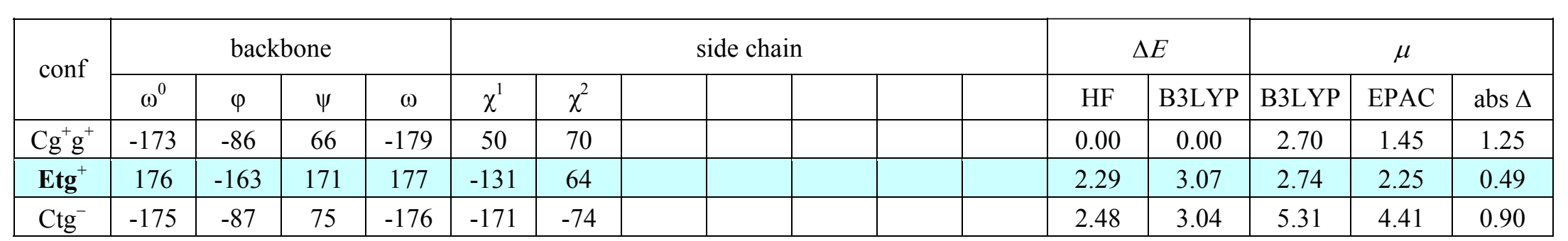




\section{(10) Ac-His(ع)-NHMe}

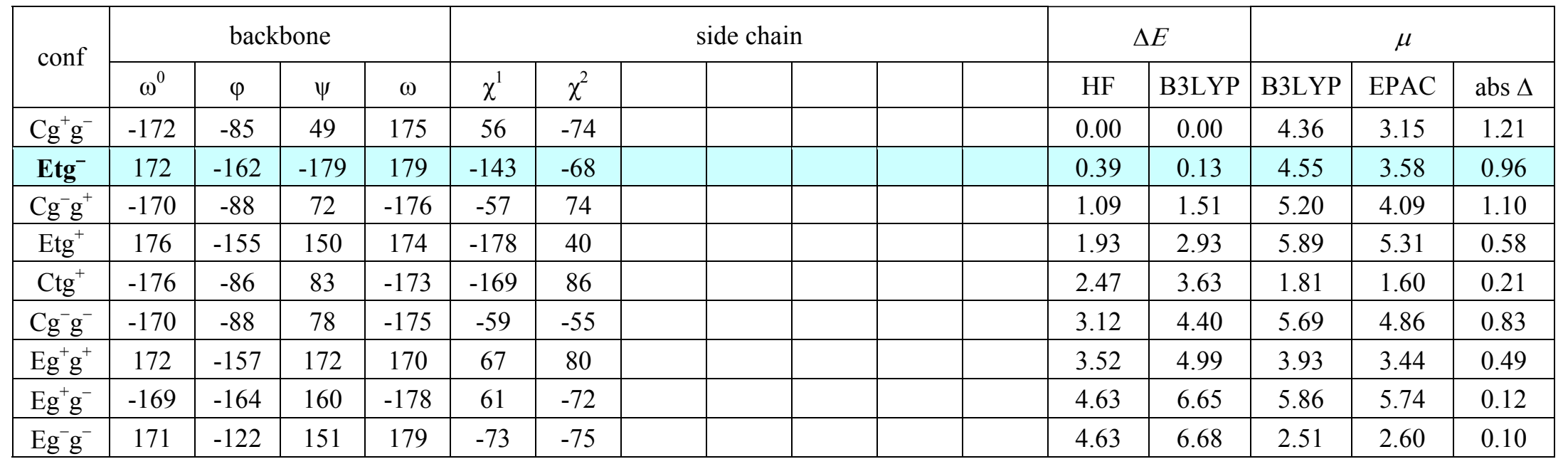

(11) Ac-Phe-NHMe

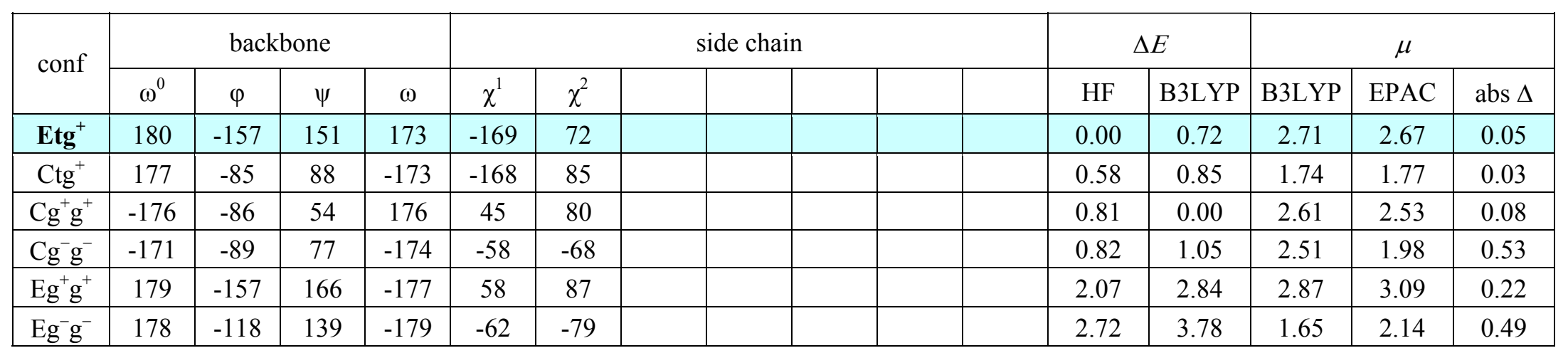


(12) Ac-Tyr-NHMe

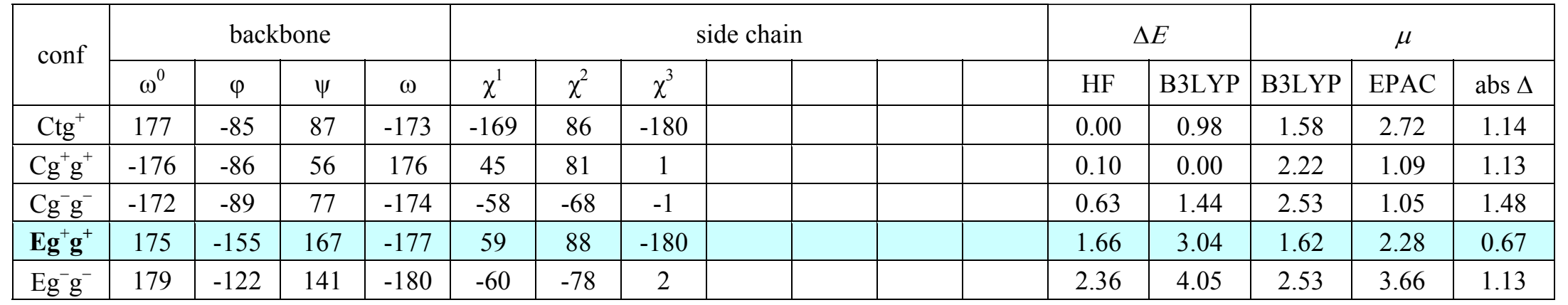

(13) Ac-Trp-NHMe

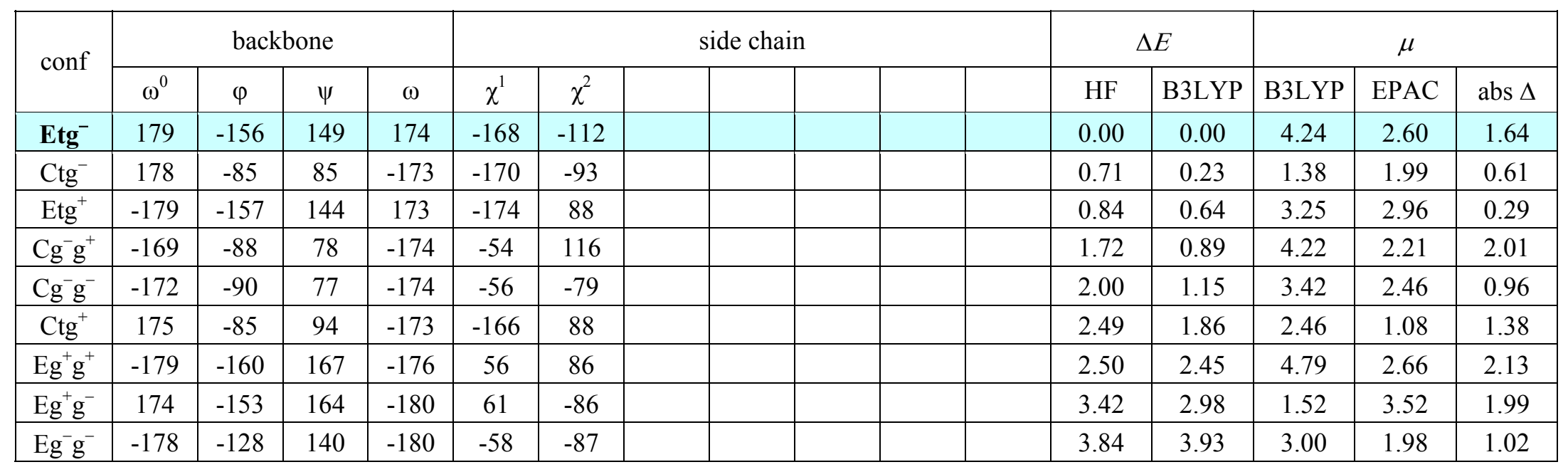


(14) Ac-Asn-NHMe

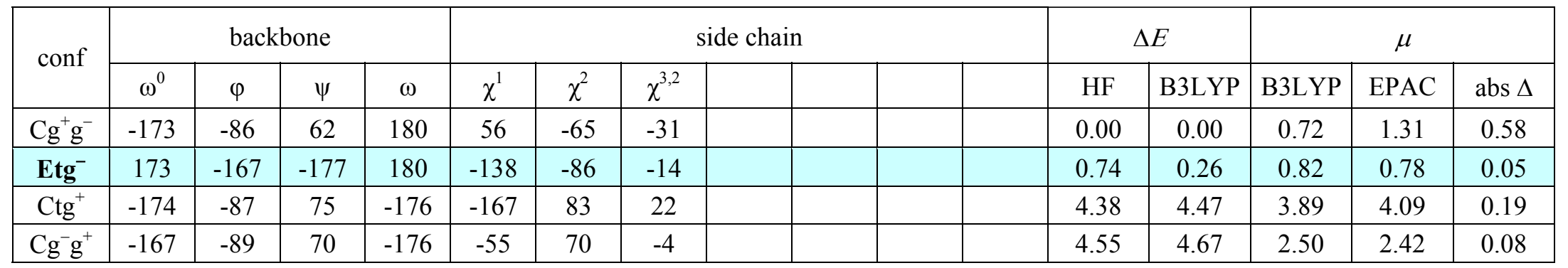

(15) Ac-GIn-NHMe

\begin{tabular}{|c|c|c|c|c|c|c|c|c|c|c|c|c|c|}
\hline \multirow{2}{*}{ conf } & \multicolumn{4}{|c|}{ backbone } & \multicolumn{6}{|c|}{ side chain } & \multicolumn{3}{|c|}{$\mu$} \\
\hline & $\omega^{0}$ & $\varphi$ & $\psi$ & $\omega$ & $\chi^{1}$ & $\chi^{2}$ & $x^{3}$ & $\chi^{4,2}$ & $\mathrm{HF}$ & B3LYP & B3LYP & EPAC & abs $\Delta$ \\
\hline $\mathbf{E g}^{-} \mathbf{g}^{-}$ & 172 & -155 & 167 & 178 & -100 & -68 & -6 & -11 & 0.00 & 0.00 & 2.88 & 2.51 & 0.37 \\
\hline $\operatorname{Etg}^{+}$ & 176 & -159 & 154 & 179 & -175 & 55 & -86 & -9 & 1.10 & 0.82 & 2.84 & 2.97 & 0.13 \\
\hline $\mathrm{Ctt}$ & -174 & -87 & 74 & -176 & -172 & -143 & -109 & -4 & 3.62 & 3.38 & 4.99 & 3.94 & 1.05 \\
\hline $\mathrm{Eg}^{+} \mathrm{g}^{+}$ & 174 & -155 & 152 & 168 & 63 & 83 & 23 & 21 & 3.95 & 4.80 & 2.74 & 2.70 & 0.04 \\
\hline $\mathrm{Eg}^{-} \mathrm{t}$ & 169 & -138 & 159 & 175 & -62 & -172 & 15 & -6 & 4.74 & 6.20 & 1.02 & 1.23 & 0.20 \\
\hline
\end{tabular}

\section{(16) Ac-Ser-NHMe}

\begin{tabular}{|c|c|c|c|c|c|c|c|c|c|c|c|c|}
\hline \multirow{2}{*}{ conf } & \multicolumn{4}{|c|}{ backbone } & \multicolumn{3}{|r|}{ side chain } & \multicolumn{2}{|c|}{$\Delta E$} & \multicolumn{3}{|c|}{$\mu$} \\
\hline & $\omega^{0}$ & $\varphi$ & $\psi$ & $\omega$ & $\chi^{1}$ & $\chi^{2}$ & & $\mathrm{HF}$ & B3LYP & B3LYP & EPAC & abs $\Delta$ \\
\hline $\mathrm{Cg}^{+} \mathrm{g}^{+}$ & -176 & -85 & 75 & -176 & 54 & 68 & & 0.00 & 0.00 & 1.53 & 1.21 & 0.32 \\
\hline Etg $^{+}$ & 170 & -157 & -176 & 178 & -166 & 82 & & 3.26 & 3.96 & 1.68 & 1.48 & 0.20 \\
\hline $\mathrm{Ctg}^{-}$ & -175 & -87 & 68 & -179 & -179 & -70 & & 3.41 & 3.96 & 3.48 & 3.88 & 0.40 \\
\hline Ett & 170 & -156 & 179 & 178 & -170 & 168 & & 4.07 & 5.30 & 2.96 & 2.86 & 0.10 \\
\hline $\mathrm{Eg}^{-} \mathrm{g}^{+}$ & 176 & -172 & 167 & -179 & -93 & 54 & & 4.64 & 4.35 & 3.13 & 3.44 & 0.31 \\
\hline
\end{tabular}




\section{(17) Ac-Thr-NHMe}

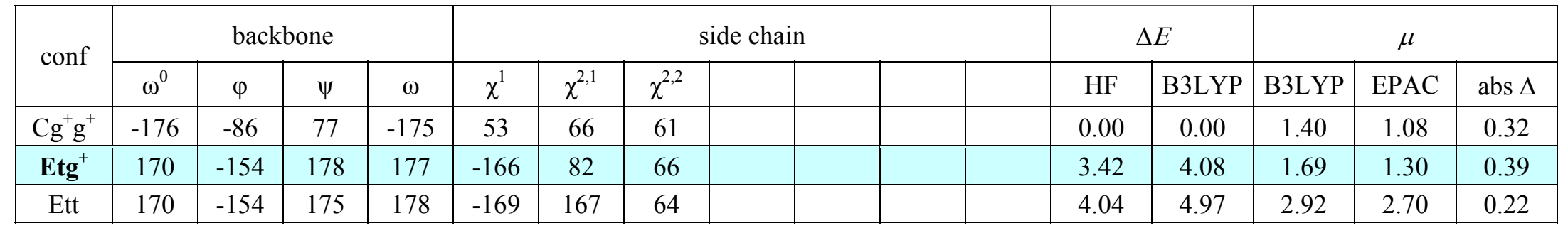

(18) Ac-Lys-NHMe

\begin{tabular}{|c|c|c|c|c|c|c|c|c|c|c|c|c|c|c|}
\hline \multirow{2}{*}{ conf } & \multicolumn{4}{|c|}{ backbone } & \multicolumn{7}{|c|}{ side chain } & \multicolumn{3}{|c|}{$\mu$} \\
\hline & $\omega^{0}$ & $\varphi$ & $\psi$ & $\omega$ & $\chi^{1}$ & $\chi^{2}$ & $\chi^{3}$ & $\chi^{4}$ & $\chi^{5}$ & $\mathrm{HF}$ & B3LYP & B3LYP & EPAC & $\operatorname{abs} \Delta$ \\
\hline $\mathrm{Ctt}$ & 179 & -86 & 82 & -173 & -172 & 177 & 179 & -180 & 174 & 0.00 & 0.00 & 1.97 & 2.02 & 0.05 \\
\hline $\mathrm{Cg}^{-} \mathrm{t}$ & 179 & -86 & 81 & -174 & -65 & -178 & -180 & -178 & 175 & 0.43 & 0.51 & 3.33 & 3.03 & 0.30 \\
\hline $\mathbf{E g}^{+} \mathbf{t}$ & 174 & -153 & 165 & -179 & 63 & -177 & 180 & -179 & 175 & 0.73 & 1.49 & 3.99 & 3.98 & 0.01 \\
\hline $\mathrm{Eg}^{-} \mathrm{t}$ & 171 & -131 & 153 & 179 & -63 & -179 & -180 & -179 & 175 & 0.85 & 1.70 & 1.39 & 1.75 & 0.36 \\
\hline Ett & -177 & -153 & 140 & 180 & -174 & 176 & -180 & -179 & 175 & 1.12 & 1.91 & 2.96 & 3.20 & 0.24 \\
\hline $\mathrm{Cg}^{-} \mathrm{g}^{-}$ & 179 & -86 & 81 & -174 & -61 & -63 & -173 & -178 & 175 & 1.21 & 1.05 & 2.31 & 2.50 & 0.19 \\
\hline $\mathrm{Cg}^{+} \mathrm{t}$ & -175 & -87 & 61 & 178 & 55 & 174 & 179 & -179 & 176 & 1.60 & 1.00 & 2.49 & 2.66 & 0.17 \\
\hline $\mathrm{Eg}^{-} \mathrm{g}^{-}$ & 171 & -133 & 152 & 179 & -66 & -72 & -180 & 177 & 172 & 1.75 & 2.29 & 2.59 & 3.07 & 0.48 \\
\hline $\mathrm{Ctg}^{-}$ & 178 & -85 & 88 & -173 & -157 & -62 & -173 & -177 & 178 & 2.14 & 1.64 & 1.98 & 2.84 & 0.86 \\
\hline $\mathrm{Eg}^{+} \mathrm{g}^{+}$ & 173 & -152 & 166 & 177 & 57 & 76 & 173 & -180 & 175 & 2.40 & 2.48 & 2.40 & 1.73 & 0.67 \\
\hline $\mathrm{Cg}^{-} \mathrm{g}^{+}$ & 179 & -86 & 79 & -174 & -78 & 68 & 171 & 179 & 173 & 2.46 & 2.13 & 2.25 & 1.11 & 1.14 \\
\hline $\operatorname{Etg}^{+}$ & -177 & -149 & 136 & -179 & -178 & 67 & 175 & -178 & 175 & 2.60 & 3.25 & 3.52 & 3.47 & 0.05 \\
\hline $\mathrm{Eg}^{+} \mathrm{g}^{-}$ & 179 & -156 & 163 & -179 & 72 & -68 & -171 & -179 & 174 & 2.93 & 3.33 & 3.24 & 4.08 & 0.85 \\
\hline $\mathrm{Eg}^{-} \mathrm{g}^{+}$ & 173 & -118 & 146 & 180 & -80 & 65 & 171 & 179 & 176 & 3.19 & 3.65 & 2.53 & 3.64 & 1.11 \\
\hline $\operatorname{Etg}^{-}$ & -177 & -151 & 135 & 179 & -175 & -89 & -179 & -178 & 177 & 3.63 & 4.01 & 2.31 & 1.67 & 0.64 \\
\hline $\mathrm{Cg}^{+} \mathrm{g}^{-}$ & -174 & -87 & 54 & 176 & 68 & -68 & -167 & -178 & 171 & 4.43 & 3.11 & 3.18 & 2.29 & 0.88 \\
\hline
\end{tabular}


(19) Ac-Arg-NHMe

\begin{tabular}{|c|c|c|c|c|c|c|c|c|c|c|c|c|c|c|c|c|}
\hline \multirow{2}{*}{ conf } & \multicolumn{4}{|c|}{ backbone } & \multicolumn{7}{|c|}{ side chain } & \multicolumn{2}{|c|}{$\Delta E$} & \multicolumn{3}{|c|}{$\mu$} \\
\hline & $\omega^{0}$ & $\varphi$ & $\psi$ & $\omega$ & $\chi^{1}$ & $\chi^{2}$ & $\chi^{3}$ & $\chi^{4}$ & $\chi^{5}$ & $\chi^{6,1}$ & $\chi^{6,2}$ & $\mathrm{HF}$ & B3LYP & B3LYP & EPAC & abs $\Delta$ \\
\hline $\operatorname{Etg}^{+}$ & 177 & -157 & 152 & 177 & -170 & 69 & -168 & 79 & 3 & 173 & -170 & 0.00 & 0.00 & 5.85 & 4.52 & 1.34 \\
\hline $\mathrm{Cg}^{+} \mathrm{g}^{+}$ & -179 & -88 & 64 & -180 & 35 & 60 & -166 & 75 & 3 & 173 & -170 & 1.44 & 0.50 & 5.37 & 2.69 & 2.67 \\
\hline $\mathrm{Eg}^{-} \mathrm{g}^{-}$ & 171 & -132 & 155 & 178 & -66 & -78 & 68 & -176 & 15 & 178 & -160 & 1.51 & 2.46 & 3.09 & 3.17 & 0.07 \\
\hline $\operatorname{Etg}^{-}$ & 177 & -156 & 152 & 176 & -160 & -92 & 170 & -74 & -7 & -175 & 169 & 2.63 & 2.21 & 6.28 & 5.25 & 1.03 \\
\hline $\mathrm{Eg}^{+} \mathrm{t}$ & 178 & -154 & 163 & -179 & 62 & -179 & 179 & -176 & 7 & 175 & -169 & 4.89 & 7.16 & 3.87 & 4.50 & 0.63 \\
\hline $\mathrm{Eg}^{-} \mathrm{t}$ & 171 & -134 & 154 & 179 & -62 & -177 & -178 & 176 & -7 & -175 & 168 & 4.92 & 7.26 & 1.26 & 2.61 & 1.35 \\
\hline
\end{tabular}

\section{(20) Ac-Asp-NHMe}

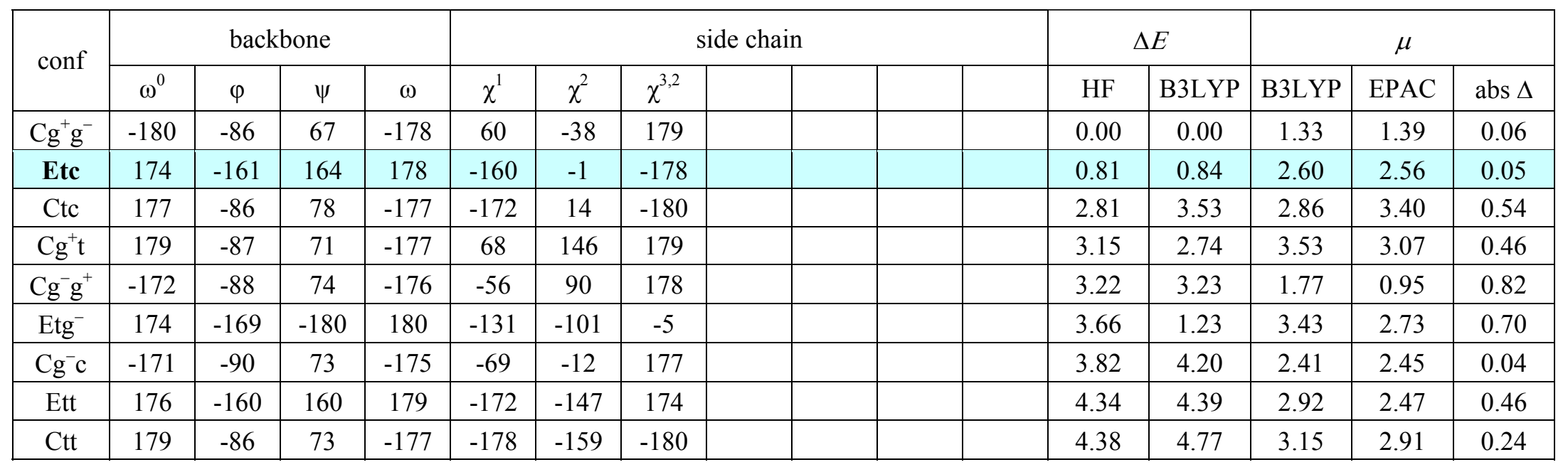


(21) Ac-Glu-NHMe

\begin{tabular}{|c|c|c|c|c|c|c|c|c|c|c|c|c|c|}
\hline \multirow{2}{*}{ conf } & \multicolumn{4}{|c|}{ backbone } & \multicolumn{6}{|c|}{ side chain } & \multicolumn{3}{|c|}{$\mu$} \\
\hline & $\omega^{0}$ & $\varphi$ & $\psi$ & $\omega$ & $\chi^{1}$ & $\chi^{2}$ & $\chi^{3}$ & $\chi^{4,2}$ & $\mathrm{HF}$ & B3LYP & B3LYP & EPAC & abs $\Delta$ \\
\hline $\mathbf{E g}^{-} \mathbf{g}^{-}$ & 172 & -153 & 165 & 178 & -99 & -70 & 4 & -179 & 0.00 & 0.16 & 2.32 & 2.25 & 0.07 \\
\hline $\mathrm{Cg}^{-} \mathrm{g}^{+}$ & -178 & -86 & 74 & -175 & -61 & 86 & -3 & -179 & 1.05 & 1.05 & 2.93 & 2.55 & 0.38 \\
\hline $\mathrm{Cg}^{+} \mathrm{g}^{-}$ & -171 & -86 & 57 & 178 & 69 & -82 & 19 & -178 & 1.18 & 0.00 & 2.63 & 2.00 & 0.64 \\
\hline $\mathrm{Etg}^{+}$ & 176 & -156 & 151 & 179 & -176 & 58 & -86 & -176 & 1.31 & 1.41 & 2.17 & 2.62 & 0.45 \\
\hline $\mathrm{Ctg}^{+}$ & -175 & -86 & 79 & -174 & -174 & 70 & 3 & -180 & 2.59 & 3.06 & 1.51 & 1.84 & 0.33 \\
\hline $\mathrm{Eg}^{+} \mathrm{g}^{+}$ & 174 & -155 & 152 & 169 & 62 & 85 & 14 & -179 & 2.74 & 3.81 & 2.10 & 1.76 & 0.34 \\
\hline $\mathrm{Cg}^{-} \mathrm{g}^{-}$ & 175 & -86 & 84 & -174 & -64 & -71 & -5 & 179 & 3.15 & 4.03 & 2.96 & 3.35 & 0.39 \\
\hline $\mathrm{Cg}^{+} \mathrm{g}^{+}$ & 175 & -88 & 64 & -180 & 39 & 51 & -85 & -173 & 3.44 & 3.12 & 1.16 & 1.59 & 0.43 \\
\hline $\mathrm{Cg}^{-} \mathrm{t}$ & 176 & -86 & 82 & -174 & -66 & 179 & -2 & -180 & 3.80 & 4.66 & 2.99 & 3.08 & 0.09 \\
\hline $\mathrm{Eg}^{-} \mathrm{g}^{+}$ & -175 & -153 & 146 & 180 & -72 & 85 & 6 & 180 & 4.08 & 5.77 & 2.91 & 3.68 & 0.77 \\
\hline $\mathrm{Ctt}$ & -179 & -86 & 80 & -174 & -174 & 173 & -133 & 178 & 4.19 & 4.90 & 3.37 & 2.66 & 0.71 \\
\hline $\mathrm{Eg}^{+} \mathrm{t}$ & 178 & -155 & 162 & -180 & 62 & -177 & 121 & -180 & 4.57 & 6.00 & 4.22 & 4.28 & 0.06 \\
\hline $\mathrm{Eg}^{-} \mathrm{t}$ & 170 & -135 & 154 & 179 & -61 & -178 & -116 & -180 & 4.69 & 6.21 & 3.97 & 3.79 & 0.17 \\
\hline
\end{tabular}

\section{(22) Ac-Lys ${ }^{+}-\mathrm{NHMe}$}

\begin{tabular}{|c|c|c|c|c|c|c|c|c|c|c|c|c|c|c|}
\hline \multirow{2}{*}{ conf } & \multicolumn{4}{|c|}{ backbone } & \multicolumn{7}{|c|}{ side chain } & \multicolumn{3}{|c|}{$\mu$} \\
\hline & $\omega^{0}$ & $\varphi$ & $\psi$ & $\omega$ & $\chi^{1}$ & $\chi^{2}$ & $\chi^{3}$ & $\chi^{4}$ & $\chi^{5}$ & $\mathrm{HF}$ & B3LYP & B3LYP & EPAC & $\operatorname{abs} \Delta$ \\
\hline $\mathrm{Eg}^{-} \mathbf{g}^{-}$ & 172 & -148 & 158 & 179 & -64 & -73 & 161 & -84 & 162 & 0.00 & 0.69 & 11.08 & 11.14 & 0.06 \\
\hline $\mathrm{Cg}^{+} \mathrm{t}$ & -170 & -86 & 58 & 179 & 55 & 149 & -94 & 62 & -179 & 0.39 & 0.00 & 10.21 & 14.20 & 3.98 \\
\hline $\mathrm{Ctg}^{-}$ & -171 & -87 & 67 & -176 & -165 & -93 & 99 & -128 & -175 & 5.55 & 5.65 & 10.16 & 12.40 & 2.24 \\
\hline $\mathrm{Eg}^{-} \mathrm{g}^{+}$ & 173 & -144 & 157 & 179 & -109 & 68 & -108 & 155 & 175 & 6.01 & 6.34 & 11.19 & 10.94 & 0.25 \\
\hline
\end{tabular}




\section{(23) Ac-Arg ${ }^{+}-\mathrm{NHMe}$}

\begin{tabular}{|c|c|c|c|c|c|c|c|c|c|c|c|c|c|c|c|c|}
\hline \multirow{2}{*}{ conf } & \multicolumn{4}{|c|}{ backbone } & \multicolumn{7}{|c|}{ side chain } & \multicolumn{2}{|c|}{$\Delta E$} & \multicolumn{3}{|c|}{$\mu$} \\
\hline & $\omega^{0}$ & $\varphi$ & $\psi$ & $\omega$ & $\chi^{1}$ & $\chi^{2}$ & $\chi^{3}$ & $\chi^{4}$ & $\chi^{5}$ & $\chi^{6,1}$ & $\chi^{6,2}$ & $\mathrm{HF}$ & B3LYP & B3LYP & EPAC & abs $\Delta$ \\
\hline $\mathbf{E g}^{-} \mathbf{g}^{-}$ & 172 & -147 & 155 & 179 & -62 & -68 & -177 & -97 & 6 & -172 & -173 & 0.00 & 0.00 & 11.99 & 12.36 & 0.37 \\
\hline $\mathrm{Eg}^{+} \mathrm{g}^{-}$ & 169 & -156 & 142 & 179 & 77 & -63 & 175 & -89 & 4 & -174 & -175 & 4.60 & 5.55 & 11.57 & 12.68 & 1.10 \\
\hline $\mathrm{Eg}^{+} \mathrm{g}^{+}$ & 174 & -154 & 133 & -180 & 58 & 96 & -172 & 87 & -2 & 174 & 176 & 4.91 & 5.98 & 12.17 & 13.23 & 1.07 \\
\hline $\mathrm{Fg}^{+} \mathrm{g}^{-}$ & -168 & -62 & 132 & -177 & 78 & -60 & 174 & -91 & 5 & -174 & -174 & 4.92 & 5.59 & 10.55 & 12.35 & 1.80 \\
\hline $\mathrm{Eg}^{-} \mathrm{g}^{+}$ & 166 & -132 & 152 & 178 & -91 & 63 & -172 & 119 & -1 & 180 & 180 & 5.90 & 4.86 & 12.05 & 12.22 & 0.17 \\
\hline $\mathrm{Ctg}^{-}$ & -175 & -87 & 68 & -176 & -163 & -76 & -170 & -178 & 0 & 179 & 179 & 9.83 & 11.17 & 14.96 & 14.57 & 0.39 \\
\hline
\end{tabular}

\section{(24) Ac-His ${ }^{+}-\mathrm{NHMe}$}

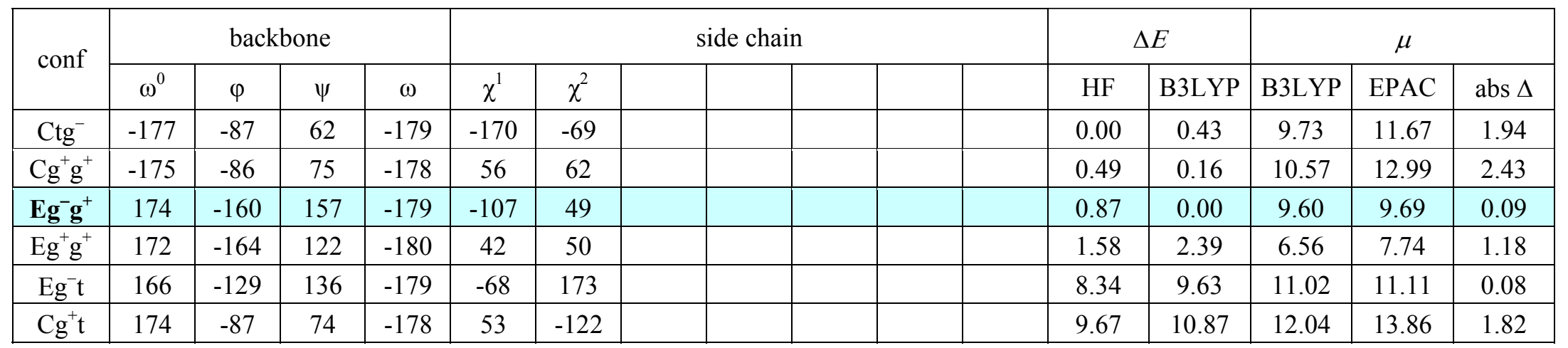




\section{(25) Ac-Asp ${ }^{-}-\mathrm{NHMe}$}

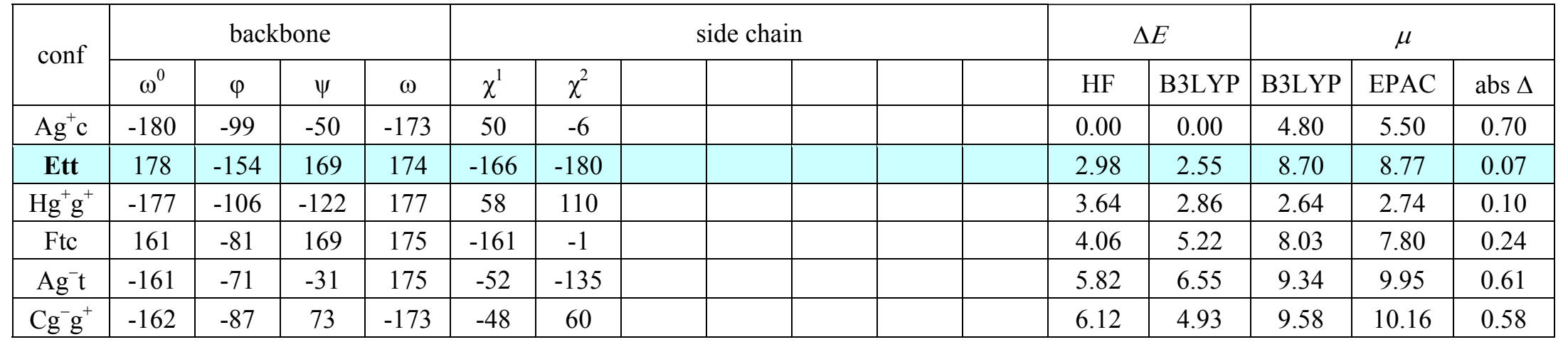

\section{(26) Ac-Glu--NHMe}

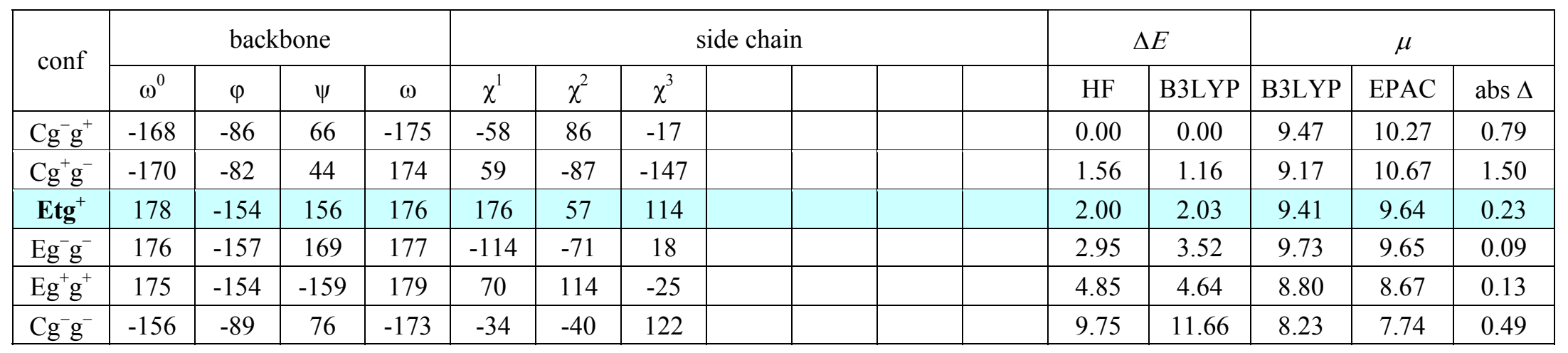

${ }^{a}$ In each Table, $\Delta E$ and $\mu$ stand for relative conformational energies in $\mathrm{kcal} / \mathrm{mol}$ and dipole moments in Debyes, respectively. Torsion angles in degrees. The absolute deviations of the dipole moments calculated by the EPAC method from those at the B3LYP/6-31G(d,p)//HF/6-31G(d,p) level are denoted by 'abs $\Delta$ '. Representative conformations used for parameterization are denoted by bold-faced letter codes. 
TABLE S5: Torsion Angles, Relative Conformational Energies, and Dipole Moments of Ac-(Ala) ${ }_{n}$-NHMe $(n=2$ to 6$)$ Calculated at the B3LYP/6-31G(d,p)//HF/6-31G(d,p) Level and Their Dipole Moments Computed by the EPAC Method ${ }^{a}$

\section{(1) Ac-(Ala) $)_{2}$-NHMe}

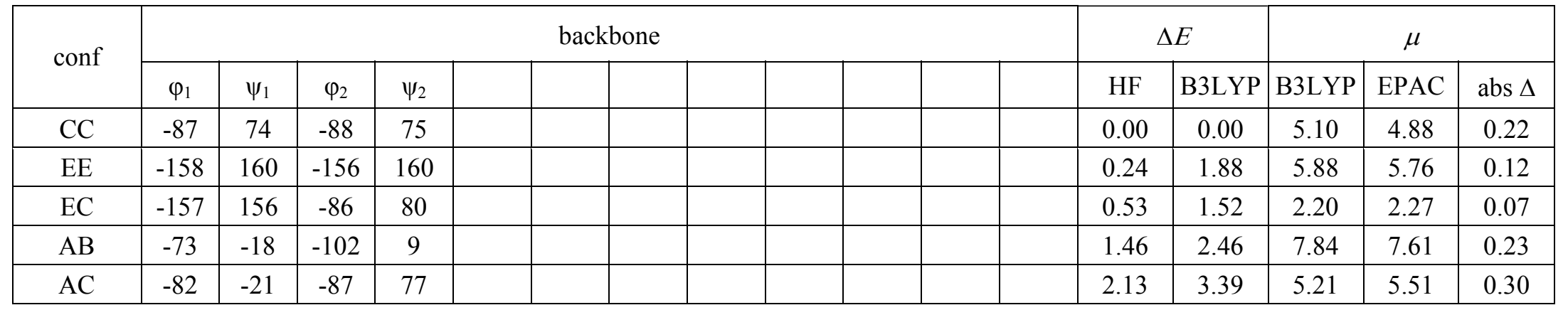

(2) Ac-(Ala) ${ }_{3}$-NHMe

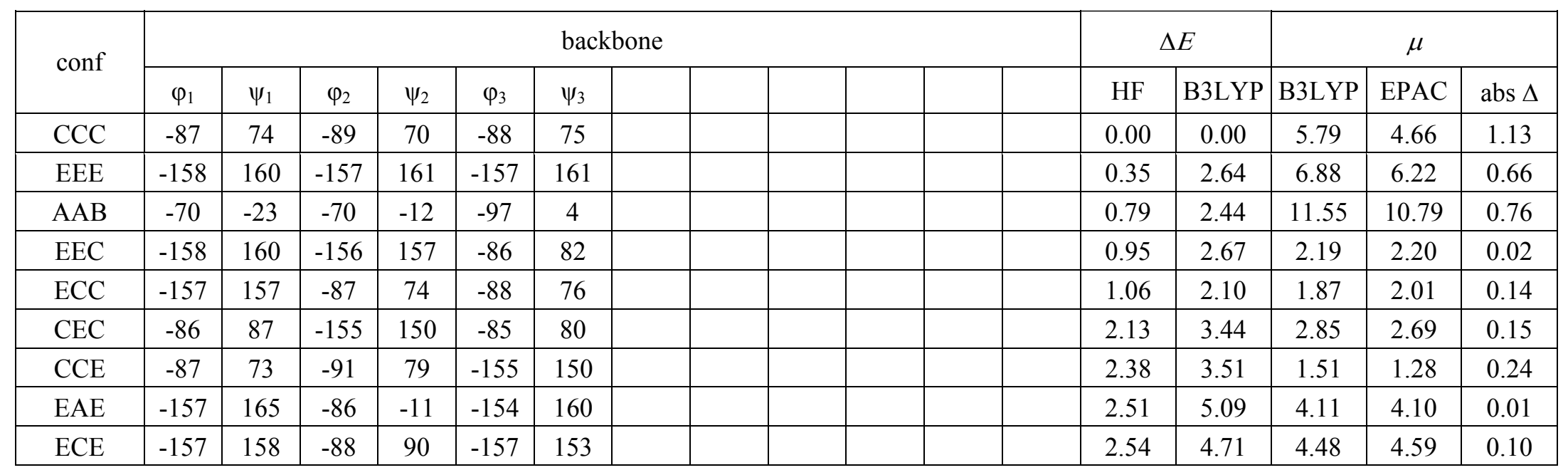




\section{(3) Ac-(Ala) ${ }_{4}$-NHMe}

\begin{tabular}{|c|c|c|c|c|c|c|c|c|c|c|c|c|c|}
\hline \multirow{2}{*}{ conf } & \multicolumn{10}{|c|}{ backbone } & \multicolumn{3}{|c|}{$\mu$} \\
\hline & $\varphi_{1}$ & $\psi_{1}$ & $\varphi_{2}$ & $\psi_{2}$ & $\varphi_{3}$ & $\psi_{3}$ & $\varphi_{4}$ & $\psi_{4}$ & $\mathrm{HF}$ & B3LYP & B3LYP & EPAC & $\operatorname{abs} \Delta$ \\
\hline AAAB & -69 & -24 & -65 & -20 & -70 & -13 & -97 & 5 & 0.00 & 1.76 & 14.83 & 13.27 & 1.56 \\
\hline $\mathrm{CCCC}$ & -87 & 74 & -88 & 70 & -89 & 70 & -88 & 74 & 0.58 & 0.00 & 8.17 & 6.87 & 1.30 \\
\hline EEEE & -158 & 160 & -157 & 161 & -157 & 161 & -157 & 161 & 1.08 & 3.41 & 9.56 & 8.61 & 0.95 \\
\hline
\end{tabular}

(4) Ac-(Ala) $)_{5}-\mathrm{NHMe}$

\begin{tabular}{|c|c|c|c|c|c|c|c|c|c|c|c|c|c|c|c|}
\hline \multirow{2}{*}{ conf } & \multicolumn{12}{|c|}{ backbone } & \multicolumn{3}{|c|}{$\mu$} \\
\hline & $\varphi_{1}$ & $\psi_{1}$ & $\varphi_{2}$ & $\psi_{2}$ & $\varphi_{3}$ & $\psi_{3}$ & $\varphi_{4}$ & $\psi_{4}$ & $\varphi_{5}$ & $\psi_{5}$ & $\mathrm{HF}$ & B3LYP & B3LYP & EPAC & $\operatorname{abs} \Delta$ \\
\hline AAAAB & -68 & -25 & -64 & -20 & -65 & -21 & -70 & -12 & -96 & 4 & 0.00 & 0.69 & 18.51 & 15.86 & 2.65 \\
\hline $\mathrm{CCCCC}$ & -87 & 74 & -88 & 70 & -88 & 70 & -89 & 70 & -88 & 74 & 2.31 & 0.00 & 9.57 & 7.38 & 2.19 \\
\hline EEEEE & -158 & 160 & -157 & 161 & -157 & 161 & -157 & 161 & -157 & 161 & 3.00 & 4.23 & 11.08 & 9.52 & 1.56 \\
\hline
\end{tabular}

\section{(5) Ac-(Ala) $)_{6}$-NHMe}

\begin{tabular}{|c|c|c|c|c|c|c|c|c|c|c|c|c|c|c|c|c|c|}
\hline \multirow{2}{*}{ conf } & \multicolumn{12}{|c|}{ backbone } & \multicolumn{2}{|c|}{$\Delta E$} & \multicolumn{3}{|c|}{$\mu$} \\
\hline & $\varphi_{1}$ & $\psi_{1}$ & $\varphi_{2}$ & $\psi_{2}$ & $\varphi_{3}$ & $\psi_{3}$ & $\varphi_{4}$ & $\psi_{4}$ & $\varphi_{5}$ & $\psi_{5}$ & $\varphi_{6}$ & $\psi_{6}$ & $\mathrm{HF}$ & B3LYP & B3LYP & EPAC & abs $\Delta$ \\
\hline AAAAAB & -68 & -25 & -64 & -21 & -64 & -21 & -65 & -20 & -70 & -13 & -96 & 4 & 0.00 & 0.00 & 22.46 & 18.79 & 3.66 \\
\hline CCCCCC & -87 & 73 & -88 & 70 & -88 & 70 & -88 & 69 & -89 & 70 & -88 & 74 & 4.24 & 0.51 & 11.75 & 9.21 & 2.54 \\
\hline EEEEEE & -158 & 160 & -157 & 161 & -157 & 161 & -157 & 161 & -157 & 162 & -157 & 161 & 5.16 & 5.58 & 13.62 & 11.71 & 1.92 \\
\hline
\end{tabular}

${ }^{a}$ In each Table, $\Delta E$ and $\mu$ stand for relative conformational energies in $\mathrm{kcal} / \mathrm{mol}$ and dipole moments in Debyes, respectively. Torsion angles in degrees. The absolute deviations of the dipole moments calculated by the EPAC method from those at the B3LYP/6-31G(d,p)//HF/6-31G(d,p) level are denoted by 'abs $\Delta$ '. 
Figure S1. Initial atomic charges for His $(\varepsilon)$, charged residues $\left(\mathrm{Lys}^{+}, \mathrm{Arg}^{+}, \mathrm{His}^{+}, \mathrm{Asp}^{-}\right.$, and $\left.\mathrm{Glu}^{-}\right)$, and some N- and C-terminal groups in electronic charges e.

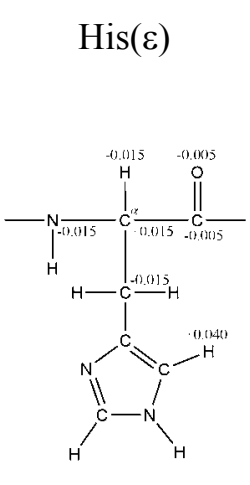

Glu

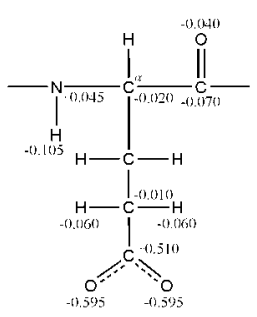

Lys $^{+}$

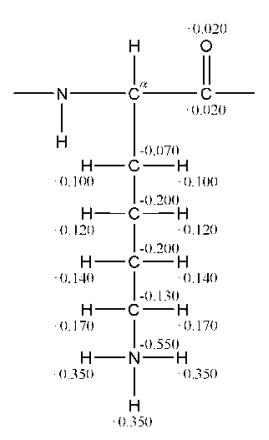

NH-

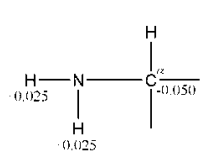

His

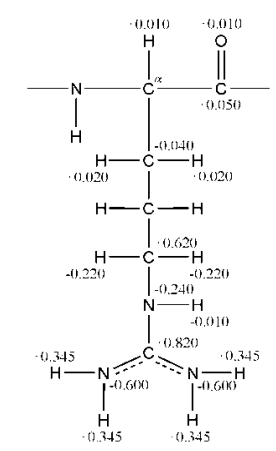

$\mathrm{H}_{2} \mathrm{~N}^{+}-$

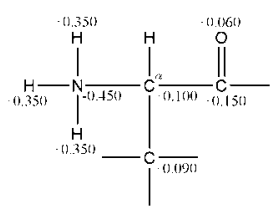

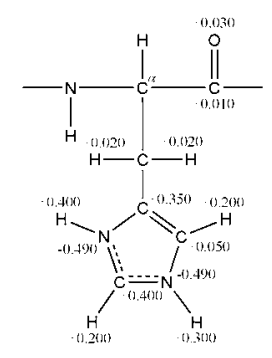

t-Boc-

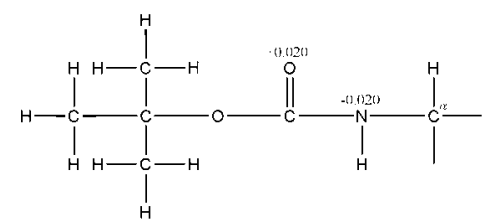

Asp

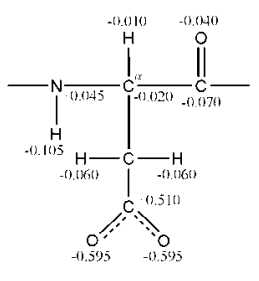

$-\mathrm{COO}^{-}$

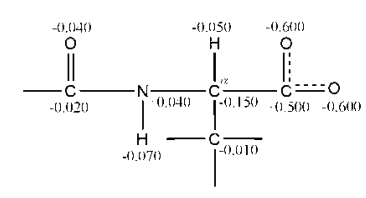

